\title{
Microwave Spectrum and Nuclear Quadrupole Interaction of 3-Bromothiophene in Excited Vibrational States
}

\author{
Yoshiaki Sasada \\ College of Engineering, Kanto Gakuin University, Kanazawa-ku, Yokohama, Japan
}

Z. Naturforsch. 38a, 1309-1319 (1983); received July 15, 1983

\begin{abstract}
The rotational spectra of 3-bromothiophene in the excited states of two vibrational modes were observed and the rotational constants, the centrifugal distortion constants, and the nuclear quadrupole coupling constants were determined. The wave numbers of the two vibrational modes were evaluated to be $210 \mathrm{~cm}^{-1}$ and $320 \mathrm{~cm}^{-1}$ by measuring relative intensities of the ground and excited vibrational transitions. Variations in the inertia defect for each of the vibrational modes are compared with the results of the approximate calculation.
\end{abstract}

\section{Introduction}

A study of the microwave spectrum of 3-bromothiophene in the ground state has been recently reported [1]. It was shown that this molecule has planar conformation with the $\mathrm{C}-\mathrm{Br}$ bond held in the plane of thiophene ring. The present study was undertaken to investigate the rotational spectrum in the excited vibrational states.

The nuclear quadrupole interaction in the molecule, due to the bromine nucleus, causes each of the rotational energy levels to split into four sublevels and consequently makes the rotational transition complicated. The analysis of the hyperfine interaction requires to use first- and secondorder perturbation theory. In cases where there exist accidental near-degeneracies in the transition energy levels, care must be taken to include the secondorder perturbation energy.

For 2-chlorothiophene [2] and 2-bromothiophene [3], a set of the satellite lines has been assigned to the excited state of an out-of-plane vibration of the carbon-halogen $(\mathrm{Cl}, \mathrm{Br})$ bond. 3-bromothiophene is also expected to have some relatively low frequency modes involving the $\mathrm{C}-\mathrm{Br}$ bond.

\section{Experimental Details}

The sample of 3-bromothiophene with $96 \%$ purity was purchased from Aldrich Chemical Company, Inc. and used without further purification. A con-

Reprint requests to Dr. Y. Sasada, Laboratory for Basic Sciences, College of Engineering, Kanto Gakuin University, Kanazawa-ku, Yokohama, 236, Japan. ventional Stark-modulated spectrometer with $100 \mathrm{kHz}$ square-wave modulation was used. The microwave spectrum was observed in the frequency region from $8.2 \mathrm{GHz}$ to $23.5 \mathrm{GHz}$. The measurements were performed between $-20^{\circ} \mathrm{C}$ and $-25^{\circ} \mathrm{C}$ with the sample pressure ranging from $15 \mathrm{mTorr}$ to $40 \mathrm{mTorr}$. The precision of the measured frequency is roughly estimated to be $0.1 \mathrm{MHz}$. The relative intensity of the lines was measured by a technique developed by Esbitt and Wilson [4].

\section{Microwave Spectrum in Excited State}

Each of the ground-state R-branch transitions corresponding to the two isotopic species of the bromine nucleus, ${ }^{79} \mathrm{Br}$ and ${ }^{81} \mathrm{Br}$, is accompanied by vibrational satellites having appreciable intensity and showing nuclear quadrupole hyperfine structure similar to that in the ground state. These spectra are assigned to a-type R-branch transitions with $\Delta K_{-1}=0$ and $\Delta K_{+1}=1$, and with $\Delta F=+1$. The assignment of the hyperfine structure is considerably facilitated by calculating hyperfine splittings using nuclear quadrupole perturbation theory. As predicted from the analysis of the spectrum in the ground state, the first-order perturbation is dominant and the second-order effects depend only on an off-diagonal element of the $e Q q$ tensor, $\chi_{a b} \mid$, though its value is very small.

Weak vibrational satellites are observed at somewhat higher frequencies than the ground-state transitions and assigned to a first excited state. In addition, very weak satellites are observed at frequencies twice as far away from the ground-state

0340-4811/ 83 / 1200-1309 \$ 01.3 0/0. - Please order a reprint rather than making your own copy. 
lines than those of the first excited state and assigned to the second excited state. These lines of the two isotopic species are listed in Table 1. The vibrational mode responsible for these two sets of satellites is designated as "mode I" for convenience.

On the other hand, the fairly weak vibrational satellites observed at lower frequencies than the ground-state transition are designated as "mode II". These assigned transitions are shown in Table 2. No other satellites are observed.

\section{Analysis and Results}

In order to determine the rotational constants and centrifugal distortion constants that yield the best fit to the hypothetical unsplit frequencies for the rotational transitions after correction for the nuclear quadrupole hyperfine splittings, a numerical method including least-squares analysis is used repeatedly. Although the centrifugal distortion effects on the rotational transitions presented by Watson [5] are estimated to be small in the excitedstate transitions as well as in the ground-state transitions, as a first step the constant, $\Delta_{J}$, among the quartic centrifugal distortion constants is included in the calculation. For the second-excited state, $\Delta_{J}$ is dropped because a better fit to the assigned transitions is obtained without it. As a result, the fitting to the theoretical transition frequencies is somewhat worse than that of the ground state. The precision of the constants in Table 3 is lower than that of the ground state. The differences between the hypothetical unsplit frequencies and the transition frequencies calculated using the constants of Table 3 are listed in Tables 1 and 2 .

As mentioned previously, the first- and secondorder perturbation energy, $E_{Q}^{(1)}$ and $E_{Q}^{(2)}$, for the nuclear quadrupole coupling effects in asymmetrictop molecules [6] can be obtained through the same procedure as described in [1] in detail. For the firstorder energy splittings

$$
\begin{aligned}
E_{\mathrm{Q}}^{(1)} & =\left\langle I, J, \tau, F, M_{\mathrm{F}}=J H_{\mathrm{Q}}^{(1)} I, J, \tau, F, M_{\mathrm{F}}=J\right\rangle \\
& =W_{a a}^{(1)} \chi_{a a}+W_{b b}^{(1)} \chi_{b b},
\end{aligned}
$$

where $H_{Q}^{(1)}$ is the first-order Hamiltonian, $\chi_{a a}=e Q\left\langle\partial^{2} V / \partial a^{2}\right\rangle, \chi_{b b}=e Q\left\langle\partial^{2} V / \partial b^{2}\right\rangle$, and $W_{a a}^{(1)}$ and $W_{b b}^{(1)}$ are the constants including the average values of the squares of the direction cosines $\left\langle J, \tau, M_{\mathrm{F}}=J \Phi_{z g}^{2} J, \tau, M_{\mathrm{F}}=J\right\rangle \quad(g=a, b, c)$, relat- ing to the space-fixed $Z$ axis. A treatment of the second-order perturbation provides the following equation including only the off-diagonal element, $\chi_{a b}$, since contributions of the other diagonal elements to $E_{Q}^{(2)}$ are very small and negligible:

$$
E_{Q}^{(2)}=W_{a b}^{(2)}\left|\chi_{a b}\right|^{2},
$$

where

$$
\begin{aligned}
& W_{a b}^{(2)}(J, \tau, F) \\
& =\sum_{J^{\prime}, \tau^{\prime}} \frac{C\left(I, J, J^{\prime}, F\right)\left[\left\langle J, \tau\left|{ }^{a} H_{a b}\right| J^{\prime}, \tau^{\prime}\right\rangle\right]^{2}}{W_{J, \tau}-W_{J^{\prime}, \tau^{\prime}}} .
\end{aligned}
$$

Here, $C\left(I, J, J^{\prime}, F\right)$ is a function of the quantum numbers and ${ }^{a} H_{a b}=\tilde{T} H_{a b} T$, where $T$ is a matrix of transformation and $H_{a b}$ is the cross product of the direction cosines, $\Phi_{z a} \Phi_{z b}$. In computating the matrix elements of (1) and (2), linear combinations of Wang's symmetric-top wave functions are employed [7]. The average values of the product of the direction cosines, then, can be computed readily by means of the procedure proposed by Schwendeman [8]. Thus, we can determine the values of $\chi_{a a}$, $\chi_{b b}$, and $\chi_{a b}$ by a least-squares fit to the observed splittings of the hyperfine components except for some of the transitions involved in the accidental near-degeneracy. The results for the two modes are listed in Table 4. The tensor components in the $\mathrm{C}-\mathrm{Br}$ bond axis system, $\chi_{z z}, \chi_{x x}$, and $\chi_{y y}$, are obtained by a rotational transformation of the $\chi$ tensor to the principal axis system. These values are also given in Table 4. The sign of the $\chi_{a b} \mid$ can not be determined from the analysis of the secondorder contribution alone. In the accidental neardegeneracy case, $W_{J \tau} \cong W_{J^{\prime} \tau^{\prime}}$, (2) is inapplicable and the following matrix is diagonalized:

$$
\left|\begin{array}{cc}
W_{J \tau}+E_{Q}^{(1)}(J, \tau) & \left\langle J, \tau\left|H_{a b}^{F}\right| J^{\prime}, \tau^{\prime}\right\rangle \\
\left\langle J^{\prime}, \tau^{\prime} H_{a b}^{F} \mid J, \tau\right\rangle & W_{J^{\prime} \tau^{\prime}}+E_{Q}^{(1)}\left(J^{\prime}, \tau^{\prime}\right)
\end{array}\right|,
$$

where $H_{a b}^{F}=\left\langle I, F, M_{F}{ }^{a} H_{a b} \mid I, F, M_{F}\right\rangle$. The correction for the levels was, however, evaluated to be very small and unimportant except for some of the transitions, $9_{18}-8_{17}$ and $9_{27}-8_{26}$ for ${ }^{79} \mathrm{Br}$ isotopic species, and $8_{26}-7_{25}, 9_{18}-8_{17}, 12_{211}-11_{210}$, and $13_{212}-12_{211}$ for ${ }^{81} \mathrm{Br}$ isotopic species, in the secondexcited vibrational state. The differences between the obseved and calculated hyperfine splittings for each of the excited states are shown in Tables 1 and 2 , respectively. 
Table 1. Observed frequencies and calculated frequencies of the quadrupole hyperfine structure of 3-bromothiophene in excited vibrational states (mode I) (in $\mathrm{MHz}$ ).

\begin{tabular}{|c|c|c|c|c|c|c|c|c|c|}
\hline \multicolumn{2}{|l|}{ Transition } & \multicolumn{4}{|c|}{${ }^{79} \mathrm{Br}$ Species } & \multicolumn{4}{|c|}{${ }^{81} \mathrm{Br}$ Species } \\
\hline$J^{\prime}-J$ & $F(\text { lower })^{\text {a }}$ & $v^{\mathrm{b}}$ & $\Delta v_{1}{ }^{\mathrm{c}}$ & $v_{\text {obs }}$ & $\Delta v_{2}{ }^{\mathrm{d}}$ & $v$ & $\Delta v_{1}$ & $v_{\text {obs }}$ & $\Delta v_{2}$ \\
\hline \multicolumn{10}{|c|}{$V=1$} \\
\hline $6_{06}-5_{05}$ & $\left.\begin{array}{r}13 / 2 \\
11 / 2 \\
9 / 2 \\
7 / 2\end{array}\right\}$ & 10508.33 & -0.04 & $\begin{array}{l}10506.22 \\
10512.48\end{array}$ & $\begin{array}{r}-0.02 \\
0.11 \\
0.00 \\
-0.06\end{array}$ & 10402.12 & -0.04 & $\begin{array}{l}10400.27 \\
10405.59\end{array}$ & $\begin{array}{r}-0.08 \\
0.02 \\
-0.06 \\
-0.11\end{array}$ \\
\hline $7_{07}-6_{06}$ & $\left.\begin{array}{r}15 / 2 \\
13 / 2 \\
11 / 2 \\
9 / 2\end{array}\right\}$ & 12238.53 & 0.06 & $\begin{array}{l}12237.07 \\
12241.58\end{array}$ & $\begin{array}{l}0.14 \\
0.26 \\
0.19 \\
0.10\end{array}$ & 12115.48 & 0.05 & $\begin{array}{l}12114.22 \\
12117.94\end{array}$ & $\begin{array}{r}0.08 \\
0.18 \\
0.03 \\
-0.04\end{array}$ \\
\hline $7_{16}-6_{15}$ & $\begin{array}{r}15 / 2 \\
13 / 2 \\
11 / 2 \\
9 / 2\end{array}$ & & & & & 12544.65 & 0.02 & $\begin{array}{l}12542.64 \\
12544.49 \\
12547.64 \\
12545.76\end{array}$ & $\begin{array}{r}0.13 \\
-0.09 \\
-0.11 \\
0.09\end{array}$ \\
\hline $8_{08}-7_{07}$ & $\left.\begin{array}{l}17 / 2 \\
15 / 2 \\
13 / 2 \\
11 / 2\end{array}\right\}$ & & & & & 13819.73 & 0.01 & $\begin{array}{l}13818.71 \\
13821.54\end{array}$ & $\begin{array}{r}0.03 \\
0.13 \\
0.05 \\
-0.05\end{array}$ \\
\hline $8_{17}-7_{16}$ & $\begin{array}{l}17 / 2 \\
15 / 2 \\
13 / 2 \\
11 / 2\end{array}$ & 14481.17 & -0.07 & $\begin{array}{l}14479.24 \\
14480.71 \\
14483.87 \\
14482.27\end{array}$ & $\begin{array}{r}-0.03 \\
-0.05 \\
0.12 \\
0.06\end{array}$ & 14329.31 & 0.08 & $\begin{array}{l}14327.73 \\
14329.04 \\
14331.52 \\
14330.05\end{array}$ & $\begin{array}{r}0.03 \\
-0.02 \\
-0.06 \\
-0.15\end{array}$ \\
\hline $8_{26}-7_{25}$ & $\begin{array}{l}17 / 2 \\
15 / 2 \\
13 / 2 \\
11 / 2\end{array}$ & 14178.83 & 0.06 & $\begin{array}{ll}14 & 175.31 \\
14 & 181.74 \\
14 & 183.28 \\
14 & 176.48\end{array}$ & $\begin{array}{r}0.15 \\
0.04 \\
-0.11 \\
-0.02\end{array}$ & 14030.21 & -0.05 & $\begin{array}{l}14026.93 \\
14032.69 \\
14027.93\end{array}$ & $\begin{array}{r}-0.09 \\
0.05 \\
-0.30\end{array}$ \\
\hline $8_{35}-7_{34}$ & $\begin{array}{l}17 / 2 \\
13 / 2 \\
11 / 2\end{array}$ & 14096.48 & -0.05 & $\begin{array}{l}14089.30 \\
14103.53\end{array}$ & $\begin{array}{r}-0.13 \\
0.06\end{array}$ & 13951.34 & 0.00 & $\begin{array}{l}13957.27 \\
13944.64\end{array}$ & $\begin{array}{l}-0.05 \\
-0.07\end{array}$ \\
\hline $9_{09}-8_{08}$ & $\left.\begin{array}{l}19 / 2 \\
17 / 2 \\
15 / 2 \\
13 / 2\end{array}\right\}$ & 15669.87 & 0.06 & 15669.05 & $\begin{array}{l}0.16 \\
0.03\end{array}$ & 15514.22 & 0.00 & $\begin{array}{l}15513.36 \\
15515.51\end{array}$ & $\begin{array}{r}-0.03 \\
0.08 \\
-0.04 \\
-0.15\end{array}$ \\
\hline $9_{19}-8_{18}$ & $\begin{array}{l}19 / 2 \\
17 / 2\end{array}$ & 15310.85 & 0.06 & $\begin{array}{l}15309.35 \\
15310.85\end{array}$ & $\begin{array}{r}-0.03 \\
0.35\end{array}$ & & & & \\
\hline $9_{18}-8_{17}$ & $\begin{array}{l}19 / 2 \\
17 / 2 \\
15 / 2 \\
13 / 2\end{array}$ & 16281.08 & -0.07 & $\begin{array}{l}16279.68 \\
16280.62 \\
16283.03 \\
16281.78\end{array}$ & $\begin{array}{r}0.08 \\
0.07 \\
-0.08 \\
-0.01\end{array}$ & 16110.55 & -0.04 & $\begin{array}{ll}16 & 109.47 \\
16 & 109.93 \\
16 & 112.19 \\
16 & 111.25\end{array}$ & $\begin{array}{r}0.18 \\
-0.24 \\
-0.08 \\
0.00\end{array}$ \\
\hline $9_{28}-8_{27}$ & $\begin{array}{l}19 / 2 \\
17 / 2 \\
13 / 2\end{array}$ & 15811.68 & 0.01 & $\begin{array}{l}15808.72 \\
15813.61 \\
15810.30\end{array}$ & $\begin{array}{r}-0.13 \\
0.17 \\
0.00\end{array}$ & & & & \\
\hline $9_{27}-8_{26}$ & $\begin{array}{l}19 / 2 \\
17 / 2\end{array}$ & 15979.19 & -0.09 & $\begin{array}{l}15976.07 \\
15981.10\end{array}$ & $\begin{array}{l}0.05 \\
0.09\end{array}$ & 15811.05 & -0.07 & $\begin{array}{l}15808.72 \\
15812.61\end{array}$ & $\begin{array}{l}0.26 \\
0.02\end{array}$ \\
\hline
\end{tabular}


Table 1 (continued)

\begin{tabular}{|c|c|c|c|c|c|c|c|c|c|}
\hline \multicolumn{2}{|l|}{ Transition } & \multicolumn{4}{|c|}{${ }^{79} \mathrm{Br}$ Species } & \multicolumn{4}{|c|}{${ }^{81} \mathrm{Br}$ Species } \\
\hline$J^{\prime}-J$ & $F{\text { (lower })^{\mathrm{a}}}$ & $v^{\mathrm{b}}$ & $\Delta v_{1}{ }^{\mathrm{c}}$ & $v_{\text {obs }}$ & $\Delta v_{2}{ }^{\mathrm{d}}$ & $v$ & $\Delta v_{1}$ & $v_{\text {obs }}$ & $\Delta v_{2}$ \\
\hline \multirow{4}{*}{$9_{37}-8_{36}$} & $\begin{array}{l}15 / 2 \\
13 / 2\end{array}$ & & & $\begin{array}{l}15982.10 \\
15977.68\end{array}$ & $\begin{array}{r}0.03 \\
-0.02\end{array}$ & & & 15813.61 & -0.05 \\
\hline & & 15858.80 & 0.03 & & & 15695.61 & 0.10 & & \\
\hline & $\left.\begin{array}{l}19 / 2 \\
13 / 2\end{array}\right\}$ & & & & & & & 15691.13 & $\begin{array}{r}-0.15 \\
0.32\end{array}$ \\
\hline & $\left.\begin{array}{l}17 / 2 \\
15 / 2\end{array}\right\}$ & & & 15863.93 & $\begin{array}{l}-0.12 \\
-0.13\end{array}$ & & & 15700.43 & $\begin{array}{r}0.32 \\
-0.15\end{array}$ \\
\hline \multirow[t]{3}{*}{$9_{36}-8_{35}$} & & 15864.24 & 0.00 & & & & & & \\
\hline & $\left.\begin{array}{l}19 / 2 \\
13 / 2\end{array}\right\}$ & & & 15859.08 & $\begin{array}{r}-0.05 \\
0.05\end{array}$ & & & & \\
\hline & $\left.\begin{array}{l}17 / 2 \\
15 / 2\end{array}\right\}$ & & & 15869.60 & $\begin{array}{r}0.05 \\
-0.05\end{array}$ & & & & \\
\hline \multirow[t]{4}{*}{$10_{110}-9_{19}$} & & 17002.21 & 0.01 & & & 16835.07 & 0.04 & & \\
\hline & $21 / 2$ & & & 17002,18 & & & & & 0.18 \\
\hline & $17 / 2$ & & & 17003.41 & $\begin{array}{r}0.34 \\
-0.37\end{array}$ & & & $\begin{array}{l}10834.94 \\
16836.36\end{array}$ & $\begin{array}{r}0.19 \\
-0.05\end{array}$ \\
\hline & $15 / 2$ & & & & & & & 16835.83 & 0.11 \\
\hline \multirow{3}{*}{$10_{19}-9_{18}$} & $21 / 2$ & & & & & 17888.13 & -0.03 & 178 & 0.11 \\
\hline & $19 / 2$ & & & & & & & 17887.73 & -0.21 \\
\hline & $17 / 2$ & & & & & & & 17889.24 & -0.24 \\
\hline \multirow[t]{4}{*}{$10_{29}-9_{28}$} & & 17560.97 & 0.02 & & & 17381.99 & -0.02 & & \\
\hline & $\begin{array}{l}21 / 2 \\
19 / 2\end{array}$ & & & $\begin{array}{l}17558.89 \\
17562.27\end{array}$ & $\begin{array}{l}0.11 \\
0.16\end{array}$ & & & $\begin{array}{l}17380.21 \\
17382.81\end{array}$ & $\begin{array}{r}0.08 \\
-0.15\end{array}$ \\
\hline & $17 / 2$ & & & 17563.48 & -0.08 & & & 17384.00 & -0.16 \\
\hline & $15 / 2$ & & & 17559.88 & -0.32 & & & & \\
\hline \multirow[t]{4}{*}{$10_{28}-9_{27}$} & & 17788.23 & -0.01 & & & 17600.14 & -0.02 & & \\
\hline & $\begin{array}{l}21 / 2 \\
19 / 2\end{array}$ & & & $\begin{array}{l}17786.02 \\
17789.59\end{array}$ & $\begin{array}{l}0.01 \\
0.15\end{array}$ & & & 17601.21 & 0.04 \\
\hline & $17 / 2$ & & & 17791.05 & 0.08 & & & 17602.34 & -0.06 \\
\hline & $15 / 2$ & & & 17787.24 & -0.19 & & & & \\
\hline \multirow[t]{2}{*}{$10_{38}-3_{37}$} & & 17625.02 & 0.09 & & & 17443.45 & 0.05 & & \\
\hline & $\begin{array}{l}21 / 2 \\
19 / 2\end{array}$ & & & $\begin{array}{l}17621.32 \\
17628.87\end{array}$ & $\begin{array}{l}0.19 \\
0.17\end{array}$ & & & $\begin{array}{l}17439.92 \\
17446.86\end{array}$ & $\begin{array}{r}-0.23 \\
0.29\end{array}$ \\
\hline \multirow[t]{3}{*}{$10_{37}-9_{36}$} & & 17634.28 & -0.01 & & & 17452.19 & 0.01 & & \\
\hline & $\begin{array}{l}21 / 2 \\
19 / 2\end{array}$ & & & 17630.29 & -0.09 & & & $\begin{array}{l}17448.86 \\
17455.49\end{array}$ & $\begin{array}{r}-0.02 \\
0.17\end{array}$ \\
\hline & $17 / 2$ & & & 17638.29 & -0.09 & & & & \\
\hline \multirow{3}{*}{$11_{011}-10_{010}$} & $23 / 21$ & & & & & $188 / 2.19$ & -0.10 & & \\
\hline & $21 / 2\}$ & & & & & & & 18871.58 & 0.07 \\
\hline & $\left.\begin{array}{l}19 / 2 \\
17 / 2\end{array}\right\}$ & & & & & & & 18873.01 & $\begin{array}{l}-0.01 \\
-0.12\end{array}$ \\
\hline \multirow[t]{4}{*}{$11_{111}-10_{110}$} & & 18690.72 & -0.20 & & & 18507.50 & 0.02 & & \\
\hline & $\left.\begin{array}{l}23 / 2 \\
21 / 2\end{array}\right\}$ & & & 18689.80 & $\begin{array}{r}0.04 \\
-0.01\end{array}$ & & & $\begin{array}{l}18506.74 \\
18507.11\end{array}$ & $\begin{array}{r}0.06 \\
-0.08\end{array}$ \\
\hline & $19 / 2\}$ & & & 10090.34 & & & & 18508.57 & 0.00 \\
\hline & $17 / 2\}$ & & & 18691.40 & 0.02 & & & 18508.17 & 0.11 \\
\hline \multirow[t]{3}{*}{$11_{110}-10_{19}$} & & & & & & 19661.39 & 0.03 & & \\
\hline & $\begin{array}{l}23 / 2 \\
21 / 2\end{array}$ & & & & & & & 19660.92 & $\begin{array}{r}0.22 \\
-0.24\end{array}$ \\
\hline & $\begin{array}{l}19 / 2 \\
17 / 2\end{array}$ & & & & & & & 19662.44 & $\begin{array}{r}-0.14 \\
0.35\end{array}$ \\
\hline \multirow[t]{4}{*}{$11_{210}-10_{29}$} & & 19307.87 & 0.00 & & & 19111.33 & -0.08 & & \\
\hline & $\begin{array}{l}23 / 2 \\
21 / 2\end{array}$ & & & 19306.08 & -0.07 & & & 19109.82 & -0.04 \\
\hline & $\begin{array}{l}21 / 2 \\
19 / 2\end{array}$ & & & $\begin{array}{l}19308.56 \\
19309.64\end{array}$ & $\begin{array}{l}-0.06 \\
-0.24\end{array}$ & & & $\begin{array}{l}19111.66 \\
19113.00\end{array}$ & $\begin{array}{r}-0.31 \\
0.03\end{array}$ \\
\hline & $17 / 2$ & & & 19307.24 & -0.13 & & & 19110.08 & 0.18 \\
\hline $11_{29}-10_{28}$ & & 19605.46 & -0.01 & & & & & & \\
\hline
\end{tabular}


Table 1 (continued)

\begin{tabular}{|c|c|c|c|c|c|c|c|c|c|}
\hline \multicolumn{2}{|l|}{ Transition } & \multicolumn{4}{|c|}{${ }^{79} \mathrm{Br}$ Species } & \multicolumn{4}{|c|}{${ }^{81} \mathrm{Br}$ Species } \\
\hline$J^{\prime}-J$ & $F$ (lower $^{\text {a }}$ & $v^{\mathrm{b}}$ & $\Delta v_{1}{ }^{\mathrm{c}}$ & $v_{\text {obs }}$ & $\Delta v_{2}{ }^{\mathrm{d}}$ & $v$ & $\Delta v_{1}$ & $v_{\text {obs }}$ & $\Delta v_{2}$ \\
\hline & $\begin{array}{l}23 / 2 \\
21 / 2 \\
19 / 2 \\
17 / 2\end{array}$ & & & $\begin{array}{l}19603.66 \\
19606.36 \\
19607.58 \\
19604.87\end{array}$ & $\begin{array}{r}0.00 \\
0.07 \\
0.05 \\
-0.02\end{array}$ & & & & \\
\hline $11_{39}-10_{38}$ & $\begin{array}{l}23 / 2 \\
21 / 2 \\
19 / 2\end{array}$ & 19392.06 & 0.09 & $\begin{array}{l}19389.14 \\
19394.91 \\
19395.44\end{array}$ & $\begin{array}{l}0.13 \\
0.21 \\
0.16\end{array}$ & 19192.06 & -0.09 & $\begin{array}{l}19189.50 \\
19194.10\end{array}$ & $\begin{array}{r}0.02 \\
-0.20\end{array}$ \\
\hline $11_{38}-10_{37}$ & $\begin{array}{l}23 / 2 \\
21 / 2 \\
19 / 2 \\
17 / 2\end{array}$ & & & & & 19206.35 & -0.01 & $\begin{array}{ll}19 & 203.91 \\
19 & 208.42 \\
19 & 209.27 \\
19 & 204.53\end{array}$ & $\begin{array}{r}0.15 \\
-0.18 \\
0.18 \\
0.28\end{array}$ \\
\hline $12_{012}-11_{011}$ & $\left.\begin{array}{l}25 / 2 \\
23 / 2 \\
21 / 2 \\
19 / 2\end{array}\right\}$ & 20737.95 & 0.03 & 20737.37 & $\begin{array}{r}-0.03 \\
0.11 \\
0.22 \\
0.08\end{array}$ & 20536.07 & 0.02 & 20535.61 & $\begin{array}{r}-0.01 \\
0.12 \\
0.13 \\
0.01\end{array}$ \\
\hline $12_{112}-11_{111}$ & $\left.\begin{array}{l}25 / 2 \\
23 / 2 \\
21 / 2 \\
19 / 2\end{array}\right\}$ & & & & & 20177.25 & -0.01 & $\begin{array}{l}20176.69 \\
20177.84\end{array}$ & $\begin{array}{r}0.12 \\
-0.26 \\
-0.26 \\
0.11\end{array}$ \\
\hline $12_{111}-11_{110}$ & $\begin{array}{l}25 / 2 \\
21 / 2 \\
19 / 2\end{array}$ & 21654.44 & -0.06 & $\begin{array}{l}21653.77 \\
21655.41\end{array}$ & $\begin{array}{r}0.12 \\
-0.06\end{array}$ & 21429.51 & 0.00 & 21428.97 & $\begin{array}{l}0.13 \\
0.06\end{array}$ \\
\hline $12_{211}-11_{210}$ & $\begin{array}{l}25 / 2 \\
23 / 2 \\
21 / 2 \\
19 / 2\end{array}$ & 21052.24 & 0.05 & $\begin{array}{ll}21 & 050.94 \\
21 & 052.84 \\
21 & 053.94 \\
21 & 051.87\end{array}$ & $\begin{array}{r}0.03 \\
0.10 \\
0.01 \\
-0.07\end{array}$ & 20838.30 & -0.03 & $\begin{array}{l}20837.05 \\
20838.65 \\
20839.62 \\
20838.07\end{array}$ & $\begin{array}{r}-0.09 \\
-0.07 \\
-0.08 \\
0.02\end{array}$ \\
\hline $12_{210}-11_{29}$ & $\begin{array}{l}25 / 2 \\
23 / 2 \\
21 / 2 \\
19 / 2\end{array}$ & 21430.38 & 0.04 & $\begin{array}{ll}21 & 428.97 \\
21 & 430.91 \\
21 & 432.11 \\
21 & 430.07\end{array}$ & $\begin{array}{r}0.06 \\
-0.04 \\
0.05 \\
0.07\end{array}$ & 21201.95 & 0.09 & $\begin{array}{ll}21 & 200.73 \\
21 & 202.38 \\
21 & 203.41 \\
21 & 201.50\end{array}$ & $\begin{array}{r}0.03 \\
-0.06 \\
0.04 \\
-0.14\end{array}$ \\
\hline $12_{39}-11_{38}$ & $\begin{array}{l}25 / 2 \\
23 / 2 \\
21 / 2\end{array}$ & 21183.30 & 0.03 & $\begin{array}{ll}21 & 181.10 \\
21 & 185.24 \\
21 & 185.91\end{array}$ & $\begin{array}{r}0.19 \\
-0.06 \\
-0.03\end{array}$ & 20963.77 & 0.08 & $\begin{array}{l}20961.93 \\
20965.56\end{array}$ & $\begin{array}{l}0.23 \\
0.13\end{array}$ \\
\hline $13_{013}-12_{012}$ & $\left.\begin{array}{l}27 / 2 \\
25 / 2 \\
23 / 2 \\
21 / 2\end{array}\right\}$ & 22407.27 & 0.00 & 22406.74 & $\begin{array}{l}0.07 \\
0.08 \\
0.20 \\
0.06\end{array}$ & 22190.53 & 0.10 & 22190.35 & $\begin{array}{l}0.22 \\
0.33 \\
0.26 \\
0.15\end{array}$ \\
\hline $13_{112}-12_{111}$ & $\left.\begin{array}{l}27 / 2 \\
25 / 2 \\
23 / 2 \\
21 / 2\end{array}\right\}$ & 23434.56 & 0.03 & $\begin{array}{l}23433.93 \\
23435.35\end{array}$ & $\begin{array}{r}-0.12 \\
-0.42 \\
-0.21 \\
0.11\end{array}$ & & & & \\
\hline $13_{212}-12_{211}$ & $\begin{array}{l}27 / 2 \\
25 / 2 \\
23 / 2\end{array}$ & 22793.73 & 0.03 & $\begin{array}{l}22792.54 \\
22794.04 \\
22794.80\end{array}$ & $\begin{array}{l}-0.08 \\
-0.22 \\
-0.22\end{array}$ & 22562.46 & -0.10 & $\begin{array}{l}22561.19 \\
22562.26 \\
22563.04\end{array}$ & $\begin{array}{l}-0.11 \\
-0.38 \\
-0.35\end{array}$ \\
\hline $13_{211}-12_{210}$ & $\begin{array}{l}27 / 2 \\
25 / 2 \\
23 / 2 \\
21 / 2\end{array}$ & 23261.80 & 0.02 & $\begin{array}{ll}23 & 260.49 \\
23 & 262.16 \\
23 & 263.15 \\
23 & 261.37\end{array}$ & $\begin{array}{r}-0.01 \\
0.04 \\
0.04 \\
-0.11\end{array}$ & 23012.98 & 0.00 & $\begin{array}{ll}23 & 012.02 \\
23 & 013.28 \\
23 & 014.19 \\
23 & 012.91\end{array}$ & $\begin{array}{r}0.08 \\
-0.04 \\
0.04 \\
0.14\end{array}$ \\
\hline $13_{311}-12_{310}$ & $27 / 2$ & 22928.15 & -0.08 & 22926.02 & -0.15 & 22691.85 & 0.05 & 22690.39 & 0.19 \\
\hline
\end{tabular}


Table 1 (continued)

\begin{tabular}{|c|c|c|c|c|c|c|c|c|c|}
\hline \multicolumn{2}{|l|}{ Transition } & \multicolumn{4}{|c|}{${ }^{79} \mathrm{Br}$ Species } & \multicolumn{4}{|c|}{${ }^{81} \mathrm{Br}$ Species } \\
\hline$J^{\prime}-J$ & $F(\text { lower })^{\mathrm{a}}$ & $v^{\mathrm{b}}$ & $\Delta v_{1}{ }^{\mathrm{c}}$ & $v_{\text {obs }}$ & $\Delta v_{2}^{\mathrm{d}}$ & $v$ & $\Delta v_{1}$ & $v_{\text {obs }}$ & $\Delta v_{2}$ \\
\hline \multirow[b]{2}{*}{$13_{310}-12_{39}$} & $\begin{array}{l}25 / 2 \\
23 / 2 \\
21 / 2\end{array}$ & 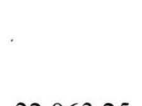 & & $\begin{array}{l}22929.52 \\
22930.38 \\
22926.88\end{array}$ & $\begin{array}{r}-0.09 \\
0.06 \\
0.02\end{array}$ & & & 22693.09 & 0.00 \\
\hline & $\begin{array}{l}27 / 2 \\
25 / 2 \\
23 / 2 \\
21 / 2\end{array}$ & 22963.25 & -0.02 & $\begin{array}{l}22961.38 \\
22964.83 \\
22965.25 \\
22961.76\end{array}$ & $\begin{array}{r}0.12 \\
0.11 \\
-0.18 \\
-0.19\end{array}$ & 22724.63 & -0.05 & $\begin{array}{l}22722.88 \\
22725.82 \\
22726.54 \\
22723.62\end{array}$ & $\begin{array}{r}-0.06 \\
-0.06 \\
0.07 \\
0.09\end{array}$ \\
\hline \multicolumn{10}{|c|}{$V=2$} \\
\hline $8_{17}-7_{16}$ & $\begin{array}{l}17 / 2 \\
15 / 2 \\
13 / 2 \\
11 / 2\end{array}$ & 14494.76 & 0.04 & $\begin{array}{l}14492.85 \\
14494.45 \\
14497.44 \\
14495.90\end{array}$ & $\begin{array}{r}-0.04 \\
0.06 \\
0.02 \\
0.16\end{array}$ & 14342.56 & -0.11 & $\begin{array}{l}14340.95 \\
14342.31 \\
14344.82 \\
14343.43\end{array}$ & $\begin{array}{r}-0.04 \\
0.06 \\
0.02 \\
0.02\end{array}$ \\
\hline $8_{26}-7_{25}$ & $\begin{array}{l}17 / 2 \\
15 / 2 \\
13 / 2\end{array}$ & & & & & 14045.35 & -0.11 & $\begin{array}{l}14044.26^{*} \\
14047.67 \\
14051.29 *\end{array}$ & $\begin{array}{l}-1.53 \\
-0.11 \\
-0.32\end{array}$ \\
\hline $9_{18}-8_{17}$ & $\left.\begin{array}{l}19 / 2 \\
17 / 2 \\
15 / 2 \\
13 / 2\end{array}\right\}$ & 16296.06 & -0.13 & $\begin{array}{l}16294.68 * \\
16298.05 \\
16296.71 *\end{array}$ & $\begin{array}{r}0.04 \\
-1.65 \\
0.02 \\
0.66\end{array}$ & 16125.57 & 0.00 & $\begin{array}{l}16124.32 \\
16122.47 * \\
16127.31\end{array}$ & $\begin{array}{r}-0.03 \\
0.45 \\
0.06\end{array}$ \\
\hline $9_{27}-8_{26}$ & $\begin{array}{l}19 / 2 \\
17 / 2 \\
15 / 2 \\
13 / 2\end{array}$ & 15996.94 & 0.06 & $\begin{array}{l}15992.74 * \\
15998.95 \\
16000.24 \\
15994.44 *\end{array}$ & $\begin{array}{l}1.18 \\
0.23 \\
0.33 \\
0.47\end{array}$ & & & & \\
\hline $\begin{array}{c}10_{19}-9_{18} \\
11_{110}-10_{19}\end{array}$ & $21 / 2$ & & & & & $\begin{array}{l}17904.63 \\
17679.36\end{array}$ & $\begin{array}{r}-0.02 \\
0.09\end{array}$ & 17903.75 & 0.09 \\
\hline & $\left.\begin{array}{l}23 / 2 \\
21 / 2 \\
19 / 2 \\
17 / 2\end{array}\right\}$ & & & & & & & $\begin{array}{l}19678.66 \\
19680.37 \\
19680.02 *\end{array}$ & $\begin{array}{r}0.09 \\
0.12 \\
-0.04 \\
0.81\end{array}$ \\
\hline $11_{29}-10_{28}$ & $\begin{array}{l}23 / 2 \\
21 / 2 \\
19 / 2 \\
17 / 2\end{array}$ & 19627.71 & 0.02 & $\begin{array}{l}19625.84 \\
19628.52 \\
19629.88 \\
19627.16\end{array}$ & $\begin{array}{r}-0.09 \\
-0.01 \\
0.09 \\
0.06\end{array}$ & & & & \\
\hline $11_{39}-10_{38}$ & $\begin{array}{l}23 / 2 \\
21 / 2 \\
17 / 2\end{array}$ & 19412.03 & -0.13 & $\begin{array}{l}19409.17 \\
19414.72 \\
19409.67\end{array}$ & $\begin{array}{l}0.15 \\
0.08 \\
0.09\end{array}$ & & & & \\
\hline $12_{111}-11_{110}$ & $\begin{array}{l}25 / 2 \\
23 / 2 \\
21 / 2 \\
19 / 2\end{array}$ & 21673.96 & 0.06 & $\begin{array}{ll}21 & 673.10 \\
21 & 673.49 \\
21 & 674.88 \\
21 & 674.58\end{array}$ & $\begin{array}{r}-0.09 \\
-0.06 \\
-0.09 \\
0.04\end{array}$ & & & & \\
\hline $12_{211}-11_{210}$ & $\left.\begin{array}{l}25 / 2 \\
23 / 2\end{array}\right\}$ & 21073.22 & 0.05 & 21071.70 * & -0.73 & 20858.84 & 0.08 & 20859.28 & $\begin{array}{l}0.11 \\
0.03\end{array}$ \\
\hline & $\begin{array}{l}21 / 2 \\
19 / 2\end{array}$ & & & $21074.94 *$ & -0.08 & & & $\begin{array}{l}20860.14 * \\
20858.60\end{array}$ & $\begin{array}{r}-0.94 \\
0.01\end{array}$ \\
\hline $12_{210}-11_{29}$ & $\begin{array}{l}23 / 2 \\
21 / 2\end{array}$ & 21454.86 & 0.00 & $\begin{array}{ll}21 & 455.42 \\
21 & 456.52\end{array}$ & $\begin{array}{l}-0.03 \\
-0.03\end{array}$ & & & & \\
\hline $13_{212}-12_{211}$ & $\begin{array}{l}27 / 2 \\
25 / 2\end{array}$ & & & & & 22584.47 & -0.08 & $\begin{array}{l}22577.00 * \\
22584.72\end{array}$ & $\begin{array}{r}-2.41 \\
0.00\end{array}$ \\
\hline
\end{tabular}


Table 1 (continued)

\begin{tabular}{|c|c|c|c|c|c|c|c|c|c|}
\hline \multicolumn{2}{|l|}{ Transition } & \multicolumn{4}{|c|}{${ }^{79} \mathrm{Br}$ Species } & \multicolumn{4}{|c|}{${ }^{81} \mathrm{Br}$ Species } \\
\hline$J^{\prime}-J$ & $F$ (lower $^{\mathrm{a}}$ & $v^{\mathrm{b}}$ & $\Delta v_{1}^{c}$ & $v_{\text {obs }}$ & $\Delta v_{2}{ }^{\mathrm{d}}$ & $v$ & $\Delta v_{1}$ & $v_{\text {obs }}$ & $\Delta v_{2}$ \\
\hline \multirow{5}{*}{$13_{211}-12_{210}$} & $\begin{array}{l}23 / 2 \\
21 / 2\end{array}$ & \multirow{5}{*}{23288.57} & \multirow{5}{*}{0.07} & & & \multirow{5}{*}{23039.62} & \multirow{5}{*}{0.03} & $\begin{array}{l}22578.38^{*} \\
22584.29\end{array}$ & $\begin{array}{l}-1.55 \\
-0.04\end{array}$ \\
\hline & & & & & & & & & \\
\hline & $\begin{array}{l}27 / 2 \\
25 / 2\end{array}$ & & & $\begin{array}{l}23287.31 \\
23288.95\end{array}$ & $\begin{array}{l}-0.03 \\
-0.03\end{array}$ & & & $\begin{array}{l}23038.54 \\
23039.96\end{array}$ & $\begin{array}{l}-0.03 \\
-0.02\end{array}$ \\
\hline & $23 / 2$ & & & 23289.99 & 0.03 & & & 23040.80 & -0.01 \\
\hline & $21 / 2$ & & & 23288.30 & 0.01 & & & & \\
\hline \multirow{4}{*}{$13_{311}-12_{310}$} & $27 / 2$ & \multirow{8}{*}{22952.22} & \multirow{8}{*}{0.07} & 22950.14 & -0.13 & & & & \\
\hline & $25 / 2$ & & & 22953.68 & 0.01 & & & & \\
\hline & $23 / 2$ & & & $22954.40 *$ & 0.10 & & & & \\
\hline & $21 / 2$ & & & 22950.82 & -0.19 & & & & \\
\hline \multirow[t]{4}{*}{$13_{310}-12_{39}$} & $27 / 2$ & & & & & 22749.03 & 0.05 & & \\
\hline & $25 / 2$ & & & & & & & $\begin{array}{l}22747.36 \\
22750.18\end{array}$ & $\begin{array}{l}-0.01 \\
-0.08\end{array}$ \\
\hline & $23 / 2$ & & & & & & & $22750.76^{*}$ & -0.60 \\
\hline & $21 / 2$ & & & & & & & $22748.02 *$ & -0.21 \\
\hline
\end{tabular}

a The transitions are $\Delta F=+1$ transitions; the lower value only is given.

b $v$ is the rotational transition frequency after the quadrupole coupling corrections.

c $\Delta v_{1}=v_{\text {obs }}-v$.

d $\Delta v_{2}$ is the difference between the observed and calculated hyperfine splittings.

e These lines are not resolved.

* These lines have been skipped in the least-squares fitting.

Table 2. Observed frequencies and calculated frequencies of the quadrupole hyperfine structure of 3-bromothiophene in excited vibrational states (mode II) (in $\mathrm{MHz}$ ).

\begin{tabular}{|c|c|c|c|c|c|c|c|c|c|}
\hline \multicolumn{2}{|l|}{ Transition } & \multicolumn{4}{|c|}{${ }^{79} \mathrm{Br}$ Species } & \multicolumn{4}{|c|}{${ }^{81} \mathrm{Br}$ Species } \\
\hline$J^{\prime}-J$ & $F(\text { lower })^{\mathrm{a}}$ & $v^{\mathrm{b}}$ & $\Delta v_{1}{ }^{\mathrm{c}}$ & $v_{\text {obs }}$ & $\Delta v_{2}^{\mathrm{d}}$ & $v$ & $\Delta v_{1}$ & $v_{\text {obs }}$ & $\Delta v_{2}$ \\
\hline \multicolumn{10}{|c|}{$V=1$} \\
\hline \multirow[t]{2}{*}{$7_{07}-6_{06}$} & & 12216.49 & 0.03 & & & 12093.43 & 0.05 & & \\
\hline & $\left.\begin{array}{l}15 / 2 \\
13 / 2\end{array}\right\}^{e}$ & & & 12214.98 & $\begin{array}{l}0.06 \\
0.21\end{array}$ & & & 12092.13 & $\begin{array}{l}0.02 \\
0.11\end{array}$ \\
\hline \multirow[t]{4}{*}{$8_{17}-7_{16}$} & & & & & & 14302.58 & -0.04 & & \\
\hline & $\begin{array}{l}17 / 2 \\
15 / 2\end{array}$ & & & & & & & $\begin{array}{l}14301.01 \\
14302.31\end{array}$ & $\begin{array}{r}0.01 \\
-0.02\end{array}$ \\
\hline & $13 / 2$ & & & & & & & 14304.77 & -0.04 \\
\hline & $11 / 2$ & & & & & 1562077 & -004 & 14303.36 & -0.08 \\
\hline $9_{28}-8_{27}$ & $\begin{array}{l}19 / 2 \\
17 / 2\end{array}$ & & & & & 15020.11 & -0.04 & $\begin{array}{l}15618.25 \\
15622.20\end{array}$ & $\begin{array}{l}-0.16 \\
-0.04\end{array}$ \\
\hline \multirow[t]{3}{*}{$9_{27}-8_{26}$} & & & & & & 15779.11 & 0.02 & & \\
\hline & $\begin{array}{l}19 / 2 \\
17 / 2\end{array}$ & & & & & & & $\begin{array}{l}15776.32 \\
15780.76\end{array}$ & $\begin{array}{r}-0.23 \\
0.15\end{array}$ \\
\hline & $15 / 2$ & & & & & & & 15778.17 & 0.24 \\
\hline \multirow[t]{3}{*}{$10_{19}-9_{18}$} & & 18043.89 & -0.02 & & & 17855.31 & -0.02 & & \\
\hline & $\begin{array}{l}21 / 2 \\
19 / 2\end{array}$ & & & $\begin{array}{l}18042.74 \\
18043.69 *\end{array}$ & $\begin{array}{l}0.03 \\
0.01\end{array}$ & & & $\begin{array}{l}17855.29 \\
17855.02\end{array}$ & $\begin{array}{l}-0.06 \\
-0.18\end{array}$ \\
\hline & $17 / 2$ & 17528.18 & -0.01 & 18045.33 & -0.15 & 1734938 & 006 & 17856.56 & 0.07 \\
\hline \multirow{4}{*}{$10_{29}-9_{28}$} & $21 / 2$ & & & 17526.04 & 0.04 & 1784.00 & 0.00 & 17347.52 & -0.02 \\
\hline & $19 / 2$ & & & 17529.34 & 0.03 & & & 17350.14 & -0.20 \\
\hline & $17 / 2$ & & & 17530.89 & 0.15 & & & 17351.34 & -0.21 \\
\hline & $15 / 2$ & & & 17527.34 & -0.07 & & & & \\
\hline
\end{tabular}


Table 2 (continued)

\begin{tabular}{|c|c|c|c|c|c|c|c|c|c|}
\hline \multicolumn{2}{|l|}{ Transition } & \multicolumn{4}{|c|}{${ }^{79} \mathrm{Br}$ Species } & \multicolumn{4}{|c|}{${ }^{81} \mathrm{Br}$ Species } \\
\hline$J^{\prime}-J$ & $F$ (lower $^{\mathrm{a}}$ & $v^{\mathrm{b}}$ & $\Delta v_{1}{ }^{\mathrm{c}}$ & $v_{\text {obs }}$ & $\Delta v_{2}{ }^{\mathrm{d}}$ & $v$ & $\Delta v_{1}$ & $v_{\text {obs }}$ & $\Delta v_{2}$ \\
\hline $10_{28}-9_{27}$ & $\begin{array}{l}21 / 2 \\
19 / 2 \\
17 / 2 \\
15 / 2\end{array}$ & 17751.87 & 0.07 & $\begin{array}{l}17749.70 \\
17753.26 \\
17754.71 \\
17750.81\end{array}$ & $\begin{array}{r}0.05 \\
0.16 \\
0.13 \\
-0.12\end{array}$ & 17564.12 & -0.04 & $\begin{array}{l}17562.27 \\
17565.06 \\
17566.34 \\
17563.48\end{array}$ & $\begin{array}{r}-0.04 \\
-0.06 \\
-0.02 \\
0.06\end{array}$ \\
\hline $11_{210}-10_{29}$ & $\begin{array}{l}23 / 2 \\
21 / 2 \\
19 / 2 \\
17 / 2\end{array}$ & 19271.95 & -0.03 & $\begin{array}{l}19270.16 \\
19272.66 \\
19274.04 \\
19271.33\end{array}$ & $\begin{array}{r}-0.07 \\
-0.04 \\
0.10 \\
-0.12\end{array}$ & & & & \\
\hline $11_{29}-10_{28}$ & $\begin{array}{l}23 / 2 \\
21 / 2 \\
19 / 2 \\
17 / 2\end{array}$ & 19564.82 & -0.10 & $\begin{array}{l}19563.18 \\
19564.47\end{array}$ & $\begin{array}{l}0.17 \\
0.24\end{array}$ & 19357.24 & 0.00 & $\begin{array}{l}19356.01 \\
19358.02 \\
19359.01 \\
19356.80\end{array}$ & $\begin{array}{l}0.25 \\
0.11 \\
0.06 \\
0.02\end{array}$ \\
\hline $12_{111}-11_{110}$ & $\left.\begin{array}{l}25 / 2 \\
23 / 2 \\
21 / 2\end{array}\right\}$ & 21615.43 & 0.01 & $\begin{array}{l}21614.83 \\
21616.47\end{array}$ & $\begin{array}{r}0.19 \\
-0.21 \\
0.01\end{array}$ & & & & \\
\hline $12_{210}-11_{29}$ & $\begin{array}{l}25 / 2 \\
23 / 2 \\
19 / 2\end{array}$ & & & $\begin{array}{ll}21 & 385.73 \\
21 & 384.39 \\
21 & 386.46 \\
21 & 385.21\end{array}$ & $\begin{array}{r}0.06 \\
0.14 \\
0.14 \\
-0.13\end{array}$ & & & & \\
\hline $13_{013}-12_{012}$ & $\left.\begin{array}{l}27 / 2 \\
25 / 2 \\
23 / 2 \\
21 / 2\end{array}\right\}$ & & & & & 22153.31 & -0.03 & $\begin{array}{l}22152.82 \\
22154.16\end{array}$ & $\begin{array}{r}-0.09 \\
0.00 \\
0.29 \\
0.20\end{array}$ \\
\hline $13_{112}-12_{111}$ & $\begin{array}{l}27 / 2 \\
25 / 2 \\
23 / 2 \\
21 / 2\end{array}$ & 23392.69 & 0.00 & $\begin{array}{l}23391.95 \\
23393.35\end{array}$ & $\begin{array}{r}-0.03 \\
-0.33 \\
-0.14 \\
0.17\end{array}$ & 23150.46 & 0.03 & $\begin{array}{l}23150.12 \\
23151.17\end{array}$ & $\begin{array}{r}0.22 \\
-0.05 \\
0.00 \\
0.28\end{array}$ \\
\hline $13_{212}-12_{211}$ & $\begin{array}{l}27 / 2 \\
25 / 2 \\
23 / 2\end{array}$ & 22751.71 & 0.02 & $\begin{array}{l}22750.38 \\
22751.83 \\
22753.15\end{array}$ & $\begin{array}{r}-0.10 \\
-0.24 \\
0.23\end{array}$ & & & & \\
\hline $13_{211}-12_{210}$ & $\begin{array}{l}27 / 2 \\
25 / 2\end{array}$ & 23213.03 & -0.02 & $\begin{array}{ll}23 & 212.03 \\
23 & 213.23\end{array}$ & $\begin{array}{r}0.23 \\
-0.22\end{array}$ & & & & \\
\hline
\end{tabular}

a The transitions are $\Delta F=+1$ transitions; the lower value only is given.

b $v$ is the rotational transition frequency after the quadrupole coupling corrections.

c $\Delta v_{1}=v_{\text {obs }}-v$.

d $\Delta v_{2}$ is the difference between the observed and calculated hyperfine splitting.

e These lines are not resolved.

* These lines are dropped out of the least-squares fitting.

Relative intensity measurements are made on the selected transitions of the ground- and first-excited vibrational states of the ${ }^{79} \mathrm{Br}$ isotopic species. Because of weakness and overlapping of the lines there are only a few transitions on which reliable measurements can be made. All measurements are carried out with the cell cooled to about $-23^{\circ} \mathrm{C}$ by a refrigerator. Care is taken to eliminate the usual sources of error, but the uncertainty is yet estimated to be about $20 \%$. The results are shown in Table 5 .

The vibrational-rotational interaction constants for the two isotopic species are given in Table 6. It must be emphasized that the values of the mode I are quite different from those of the mode II in sign and magnitude. 
Table 3. Rotational constants, moments of inertia ${ }^{c}$, and inertia defects ${ }^{d}$ of 3 -bromothiophene in the ground and excited vibrational states.

\begin{tabular}{|c|c|c|c|c|}
\hline & \multirow[b]{2}{*}{$V=0$} & \multicolumn{2}{|l|}{ mode I } & \multirow{2}{*}{$\begin{array}{l}\text { mode II } \\
V=1\end{array}$} \\
\hline & & $V=1$ & $V=2$ & \\
\hline \multicolumn{5}{|c|}{${ }^{79} \mathrm{Br}$ Species } \\
\hline $\begin{array}{ll}A & (\mathrm{MHz}) \\
B & (\mathrm{MHz}) \\
C & (\mathrm{MHz}) \\
\Delta_{J}(\mathrm{~Hz}) \\
\varkappa\end{array}$ & $\begin{array}{c}7105.11^{\mathrm{a}}(43)^{\mathrm{b}} \\
933.111 \quad(1) \\
824.611 \quad(1) \\
35.1 \quad(34) \\
-0.965449\end{array}$ & $\begin{array}{ll}7019.7 & (7) \\
934.076 & (3) \\
825.651 & (3) \\
20 & (7) \\
-0.96499\end{array}$ & $\begin{array}{l}6935.5 \quad(14) \\
934.902 \text { (6) } \\
826.623 \text { (9) } \\
-0.96455\end{array}$ & $\begin{array}{cc}7104.7 & (8) \\
932.313 & (5) \\
824.079 & (5) \\
27 & (12) \\
-0.96553\end{array}$ \\
\hline $\begin{array}{l}I_{a}\left(\mathrm{amu} \AA^{2}\right) \\
I_{b}\left(\mathrm{amu} \AA^{2}\right) \\
I_{c}\left(\mathrm{amu} \AA^{2}\right) \\
\Delta\left(\mathrm{amu} \AA^{2}\right)\end{array}$ & $\begin{array}{r}71.1289(4) \\
541.607 \quad(4) \\
612.870(5) \\
0.134 \quad(8)\end{array}$ & $\begin{array}{r}71.994 \quad(7) \\
541.047 \quad(4) \\
612.098(5) \\
-0.943 \quad(9) \\
{ }^{81} \mathrm{Br} \mathrm{Speci}\end{array}$ & $\begin{array}{r}72.868(15) \\
540.569(5) \\
611.378(8) \\
-2.059(18)\end{array}$ & $\begin{array}{r}71.133(8) \\
542.070(4) \\
613.265(6) \\
0.062(11)\end{array}$ \\
\hline $\begin{array}{l}A(\mathrm{MHz}) \\
B(\mathrm{MHz}) \\
C(\mathrm{MHz}) \\
\Delta_{J}(\mathrm{~Hz}) \\
\chi\end{array}$ & $\begin{array}{l}7103.60(44) \\
922.998(1) \\
816.700 \quad(1) \\
47.8 \quad(34) \\
-0.966184\end{array}$ & $\begin{array}{ll}7017.1 & (8) \\
923.935 & (3) \\
817.744 & (3) \\
14 & (7) \\
-0.96573\end{array}$ & $\begin{array}{l}6932.0 \quad(17) \\
924.780 \quad(5) \\
818.658 \quad(8) \\
-0.96528\end{array}$ & $\begin{array}{cc}7102.8 & (9) \\
922.209 & (4) \\
816.145 & (4) \\
14 & (10) \\
-0.96626\end{array}$ \\
\hline $\begin{array}{ll}I_{a} & \left(\mathrm{amu} \AA^{2}\right) \\
I_{b} & \left(\mathrm{amu} \AA^{2}\right) \\
I_{c} & \left(\mathrm{amu} \AA^{2}\right) \\
\Delta & \left(\mathrm{amu} \AA^{2}\right)\end{array}$ & $\begin{aligned} & 71.1440(4) \\
& 547.541(64) \\
& 618.806(5) \\
& 0.121(8)\end{aligned}$ & $\begin{array}{r}72.021 \quad(8) \\
546.986 \quad(4) \\
618.016 \quad(5) \\
-0.991(10)\end{array}$ & $\begin{array}{r}72.905(18) \\
546.486 \quad(5) \\
617.326(8) \\
-2.065(20)\end{array}$ & $\begin{array}{r}71.152(9) \\
548.009(5) \\
619.227(6) \\
0.066(12)\end{array}$ \\
\hline
\end{tabular}

a From Ref. [1].

b Uncertainties, in parentheses, are standard deviations of the fits.

c The conversion factor 505379.1 (3.8) (amu $\AA^{2} \mathrm{MHz}$ ) is used.

d $\Delta=I_{c}-I_{a}-I_{b}$.

Table 4. Quadrupole coupling constants of 3-bromothiophene in the excited vibrational states (in $\mathrm{MHz}$ ).

\begin{tabular}{|c|c|c|c|}
\hline & \multicolumn{2}{|l|}{ mode I } & \multirow{2}{*}{$\begin{array}{l}\text { mode II } \\
V=1\end{array}$} \\
\hline & $V=1$ & $V=2$ & \\
\hline \multicolumn{4}{|c|}{${ }^{79} \mathrm{Br}$ Species } \\
\hline $\begin{array}{l}\chi_{a a} \\
\chi_{b b} \\
\chi_{c c} \\
\chi_{a b} \mid\end{array}$ & $\begin{array}{r}554.5(32)^{\mathrm{a}} \\
-280.9(26) \\
-273.6(41) \\
61 \quad(27)\end{array}$ & $\begin{array}{rr}549 & (8) \\
-281 & (4) \\
-268 & (9) \\
106(119)\end{array}$ & $\begin{array}{r}554(12) \\
-282(7) \\
-272(14) \\
61(50)\end{array}$ \\
\hline $\begin{array}{l}\chi_{z z} \\
\chi_{x x} \\
\chi_{y y}\end{array}$ & $\begin{array}{r}558.9(48) \\
-285.3(44) \\
-273.6(65)\end{array}$ & $\begin{array}{r}562(30) \\
-294 \quad(30) \\
-267 \quad(42)\end{array}$ & $\begin{array}{r}559(13) \\
-287(9) \\
-272) 16)\end{array}$ \\
\hline \multicolumn{4}{|c|}{${ }^{81} \mathrm{Br}$ Species } \\
\hline $\begin{array}{l}\chi_{a a} \\
\chi_{b b} \\
\chi_{c c} \\
\chi_{a b}\end{array}$ & $\begin{array}{r}470.3(36) \\
-238.1(28) \\
-232.2(46) \\
49 \quad(32)\end{array}$ & $\begin{array}{r}461 \quad(6) \\
-238 \quad(4) \\
-223 \quad(7) \\
97(122)\end{array}$ & $\begin{array}{r}461(10) \\
-233(5) \\
228(13) \\
55(41)\end{array}$ \\
\hline $\begin{array}{l}\chi_{z z} \\
\chi_{x x} \\
\chi_{y y}\end{array}$ & $\begin{array}{r}473.7(54) \\
-241.5(49) \\
-232.2(73)\end{array}$ & $\begin{array}{r}474 \\
-251 \\
-223 \\
-232) \\
\end{array}$ & $\begin{array}{r}465(12) \\
-237(8) \\
-228(14)\end{array}$ \\
\hline
\end{tabular}

a Uncertainties, in parentheses, are standard deviations of the fits.

\section{Discussion}

Valuable information on the planarity and the vibrational modes for a molecule can be obtained from the study of the inertia defect $[9,10]$. The experimental values for the two modes are already given in Table 3. The inertia defect due to the vibrational-rotational interaction in the planar molecule can be expressed by the formula

$$
\begin{gathered}
\Delta_{v}=\sum_{s} \Delta_{s}\left(v_{s}+\frac{1}{2}\right), \\
\Delta_{s}=\frac{h}{\pi^{2} c}\left\{\sum_{s^{\prime}} \frac{\omega_{s^{\prime}}^{2}}{\omega_{s}\left(\omega_{s}^{2}-\omega_{s^{\prime}}^{2}\right)}\right. \\
\cdot\left[\left(\zeta_{s s^{\prime}}^{(\mathrm{a})}\right)^{2}+\left(\zeta_{s s^{\prime}}^{(\mathrm{b})}\right)^{2}-\left(\zeta_{s s^{\prime}}\right)^{(\mathrm{c})}\right] \\
\left.+\delta_{s t} \frac{3}{2 \omega_{t}}\right\} .
\end{gathered}
$$

Here $\omega_{s}$ and $\omega_{s^{\prime}}$ are vibrational fundamentals and the $\zeta$ 's are Coriolis coupling constants. For a planar molecule, the large negative change of the inertia defect in going from the ground to the excited 
Table 5. Relative intensities ${ }^{b}$ of the first-excited vibrational state/ground state for 3-bromothiophene $\left({ }^{79} \mathrm{Br}\right.$ species).

\begin{tabular}{|c|c|c|c|}
\hline \multicolumn{2}{|l|}{ Transition } & \multicolumn{2}{|c|}{ Ratio of intensities } \\
\hline$J^{\prime}-J$ & $F$ (lower) $^{\text {a }}$ & $\begin{array}{l}\text { mode I } \\
V=1\end{array}$ & $\begin{array}{l}\text { mode II } \\
V=1\end{array}$ \\
\hline $8_{17}-7_{16}$ & $\begin{array}{l}17 / 2 \\
15 / 2 \\
13 / 2\end{array}$ & $\begin{array}{l}0.30 \\
0.30 \\
0.28\end{array}$ & \\
\hline $9_{18}-8_{17}$ & $\begin{array}{l}19 / 2 \\
15 / 2\end{array}$ & $\begin{array}{l}0.33 \\
0.31\end{array}$ & \\
\hline $9_{27}-8_{26}$ & $\begin{array}{l}19 / 2 \\
17 / 2 \\
15 / 2\end{array}$ & $\begin{array}{l}0.29 \\
0.34 \\
0.29\end{array}$ & \\
\hline $10_{28}-9_{27}$ & $\begin{array}{l}21 / 2 \\
19 / 2 \\
17 / 2\end{array}$ & $\begin{array}{l}0.27 \\
0.29\end{array}$ & $\begin{array}{l}0.15 \\
0.18 \\
0.17\end{array}$ \\
\hline $11_{210}-10_{29}$ & $\begin{array}{l}23 / 2 \\
21 / 2\end{array}$ & 0.30 & $\begin{array}{l}0.21 \\
0.15\end{array}$ \\
\hline $11_{29}-10_{28}$ & $\begin{array}{l}23 / 2 \\
19 / 2\end{array}$ & $\begin{array}{l}0.32 \\
0.28\end{array}$ & $\begin{array}{l}0.12 \\
0.15\end{array}$ \\
\hline $12_{210}-11_{29}$ & $21 / 2$ & & 0.14 \\
\hline $\begin{array}{l}\text { Average } \\
\bar{v}\left(\mathrm{~cm}^{-1}\right)\end{array}$ & & $\begin{array}{l}0.30 \\
2.1 \times 10^{2}\end{array}$ & $\begin{array}{l}0.16 \\
3.2 \times 10^{2}\end{array}$ \\
\hline
\end{tabular}

a The transitions are $\Delta F=+1$ transitions; the lower $F$ value only is given.

b The measurements were carried out at $-20^{\circ} \mathrm{C} \sim-25^{\circ} \mathrm{C}$.

Table 6. Vibration-rotation interaction constants $^{\mathrm{b}}$ of 3-bromothiophene (in $\mathrm{MHz}$ ).

\begin{tabular}{llll}
\hline \multicolumn{3}{c}{ mode I } & \multicolumn{2}{c}{ mode II } \\
\hline \multicolumn{4}{c}{${ }^{79} \mathrm{Br}$ Species } \\
$\alpha$ & $85.4 \quad(8)^{\mathrm{a}}$ & 0.41 & $(70)$ \\
$\beta$ & $-0.965(3)$ & $0.779(4)$ \\
$\gamma$ & $-1.040(3)$ & $0.532(4)$ \\
\multicolumn{5}{c}{} \\
$\alpha$ & $86.6 \quad(9)$ & $0.80(98)$ \\
$\beta$ & $-0.937(3)$ & $0.789(4)$ \\
$\gamma$ & $-1.044(3)$ & $0.555(4)$ \\
\hline
\end{tabular}

a Uncertainties, in parentheses, are standard deviations of the fits.

$b$ The values were obtained from the equations

$$
\begin{aligned}
& A_{t}=A_{\mathrm{e}}-\alpha(v+1 / 2), \\
& B_{r}=B_{\mathrm{e}}-\beta(v+1 / 2), \\
& C_{r}=C_{\mathrm{e}}-\gamma(v+1 / 2) .
\end{aligned}
$$

$A_{0}, B_{0}$, and $C_{0}$ in [1] are used. vibrational state is, in general, typical for an out-ofplane vibration. Furthermore, the difference between the inertia defects in the two adjacent excited states, $\Delta_{t+1}-\Delta_{t}$, can also provide information on the character of the out-of-plane vibration. In the limiting case where any other vibrational mode with which the vibrational modes couple is at a much higher frequency, $\omega_{s} \ll \omega_{s^{\prime}}$, (3) is reduced to the simple form [11]

$$
\Delta_{t}=-\frac{h}{2 \pi c^{2} \omega_{t}} .
$$

Although many of the successively excited states of the mode I are not found, it is reasonable to assume that the transitions are caused by the out-ofplane vibration of the $\mathrm{C}-\mathrm{Br}$ bond. Then, from Table 3, $\Delta_{1}-\Delta_{0}=-1.077 \mathrm{amu} \AA^{2}$ for the ${ }^{79} \mathrm{Br}$ species and accordingly the value of $\omega_{t}$ is calculated to be $62.6 \mathrm{~cm}^{-1}$ from (4). The deviation from the value $210 \mathrm{~cm}^{-1}$ obtained from the relative intensity measurements is very large. It is uncertain whether these two results can be considered to be significantly different. We consider that the discrepancy comes from the interaction with the other low-frequency vibrational modes, because it can be seen that inclusion of the coupling terms in (3) results in a negative large value of $\Delta_{t}$ for $\omega_{t}<\omega_{s^{\prime}}$.

Prior to the analysis of the interaction between the vibrational modes, the Raman spectrum of 3 -bromothiophene in liquid phase was observed in the range $50 \mathrm{~cm}^{-1}$ to $500 \mathrm{~cm}^{-1}$ on a JACOM R- 800 Raman spectrometer equipped with an argon ion laser $(\lambda=5145 \AA)$. Two medium peaks at $200 \mathrm{~cm}^{-1}$ and $243 \mathrm{~cm}^{-1}$, and a strong sharp peak at $319 \mathrm{~cm}^{-1}$ are observed. Two other very weak peaks are detected at $393 \mathrm{~cm}^{-1}$ and $465 \mathrm{~cm}^{-1}$. These values are consistent with those of the infrared absorption spectrum reported in [12] except for the line $393 \mathrm{~cm}^{-1}$. The vibrational modes of 3 -substituted thiophene correspond closely to the results of the infrared and Raman spectra of 2-substituted thiophene $[13,14]$. The vibrational fundamentals of halogenothiophene is also similar to those of halogenobenzene [15]. Although a complete infrared study of 3-bromothiophene for the vibrational modes of low frequency has not been made, it may be assumed that the lowest frequency line corresponds to the out-of-plane vibration involving the bromine nucleus and also the two lines of the nextlowest frequency to the in-plane vibrations. In 
the computation of Coriolis coupling constants we consider an approximate model in which the vibrations involving strong restorring forces can be treated independently of the rest. In addition, the bromine nucleus is bound to the "frozen" ring of thiophene, where the bonds and angles are rigid and the ring is replaced with a triangle made of the three carbon nuclei closed to the bromine nucleus. In that case, we can calculate the apparent values of the ' $\zeta$ 's by use of the approximate method [16]. If the out-of-plane and in-plane vibrations in the model couple, $\left(\zeta_{t s}^{(\mathrm{c})}\right)^{2}$ in (3) is dropped, since a set of an out-of-plane vibration and an inplane vibration produces a vibrational angular momentum along the in-plane axes [17]. Thus, using the calculated $\left(\zeta_{t s_{1}^{\prime}}^{(\mathrm{a})}\right)^{2}+\left(\zeta_{t s_{1}^{\prime}}^{(\mathrm{b})}\right)^{2}=0.742$ and $\left(\zeta_{t s_{2}^{\prime}}^{(\mathrm{a})}\right)^{2}+\left(\zeta_{t s_{2}}^{(\mathrm{b})}\right)^{2}=0.131$ for the two couplings in (3) leads to $\Delta_{1}-\Delta_{0}=$ $-1.07 \mathrm{amu} \AA^{2}$. The agreement between the experimental and calculated values is quite satisfactory whereas the inertia defect is sensitive to the frequencies of the coupling vibrations.

For the mode II, the value of inertia defect is positive and very small as shown in Table 3 . If interaction is taking place in sets of two in-plane vibrations, from (3)

$\Delta_{v+1}-\Delta_{v}=\frac{h}{\pi^{2} c} \sum_{s^{\prime}}\left[\frac{\omega_{s^{\prime}}^{2}}{\omega_{s}\left(\omega_{s^{\prime}}^{2}-\omega_{s}^{2}\right)}\left(\zeta_{s^{\prime} s}^{(c)}\right)^{2}\right]$,

since interaction between two in-plane vibrations

[1] Y. Sasada, M. Saitoh, and S. Tobita, J. Mol. Spectrosc. 92, 363 (1982).

[2] J. Mjöberg and S. O. Ljunggren, Z. Naturforsch. A 28, 729 (1973).

[3] P. J. Mjöberg, W. M. Ralowski, and S. O. Ljunggren, Z. Naturforsch. A30, 541 (1975).

[4] A. S. Esbitt and E. B. Wilson, Jr., Rev. Sci. Instrum. 34, 901 (1963).

[5] J. K. G. Watson, J. Chem. Phys. 46, 1935 (1967).

[6] J. K. Bragg, Phys. Rev. 74, 533 (1948).

[7] G. W. King, R. M. Hainer, and P. C. Cross, J. Chem. Phys. 11, 27 (1943).

[8] R. H. Schwendeman, J. Mol. Spectrosc. 15, 451 (1965).

[9] T. Oka and Y. Morino, J. Mol. Spectrosc. 6, 472 (1961). produces a vibrational angular momentum only along the out-of-plane axis " $c$ ", [17]. Accordingly, we obtain $\Delta_{1}-\Delta_{0}=0.062 \mathrm{amu} \AA^{2}$ in the case of $\omega_{s}<\omega_{s^{\prime}}$ using the calculated value $\left(\zeta_{s^{\prime} s}^{(\mathrm{c})}\right)^{2}=0.048$ in (5), and its value is close to the experimental value -0.072 amu $\AA^{2}$.

This approximation would not suffice to safely estimate the inertia defect due to the vibrationalrotational interaction since it involves the assumptions discussed above, but the results show that the variations of the inertia defects have to be attributed to the appreciable interactions between the vibrational modes of the $\mathrm{C}-\mathrm{Br}$ bond.

\section{Acknowledgements}

The author wishes to acknowledge Professor T. Satoh, National Defense Academy, for continued encouragement and stimulating discussion throughout this work. He wishes also to thank Professor E. Hirota, Institute for Molecular Science, who read the manuscript and made very helpful suggestions and Dr. M. Akiyama, St. Paul's University, for the useful discussions on the analysis of the Raman spectrum. Furthermore, he is indebted to $\mathrm{Mr}$. T. Homma and his staff members in Japan Spectroscopic Co., LTD for using a Raman spectrometer. The research was supported in part by the Kanto Gakuin University Foundation.

[10] D. R. Herschbach and V. W. Laurie, J. Chem. Phys. 40, 3142 (1964).

[11] Y. Hanyu, C. O. Britt, and J. E. Boggs, J. Chem. Phys. 45, 4725 (1966).

[12] Yo. M. Kimelfeld, V. T. Aleksanyan, N. N. Magdesieva, and Yu. K. Yurev, Zh. Strukt. Khim. 7, 42 (1966).

[13] S. Gronowitz, A. R. Katritzky, and R. E. Reavill, J. Chem. Soc. 3881 (1963).

[14] M. Horak, I. J. Hyams, and E. R. Lippincott, Spectrochim. Acta 22, 1355 (1966).

[15] D. H. Whiffen, J. Chem. Soc. 1350 (1956).

[16] J. H. Meal and S. R. Polo, J. Chem. Phys. 24, 1126 (1956).

[17] K. Kuchitsu, T. Oka, and Y. Morino, J. Mol. Spectrosc. 15, 51 (1965). 